\title{
Conformational effects of Lys191 in the human GnRH receptor: mutagenesis and molecular dynamics simulations studies
}

\author{
Eduardo Jardón-Valadez ${ }^{1,2, *}$, Arturo Aguilar-Rojas ${ }^{1, *}$, Guadalupe Maya-Núñez ${ }^{1}$, \\ Alfredo Leaños-Miranda ${ }^{1}$, Ángel Piñeiro ${ }^{2,3}$, P Michael Conn $^{1,4}$ and Alfredo Ulloa-Aguirre ${ }^{1,4}$ \\ ${ }^{1}$ Research Unit in Reproductive Medicine, Hospital de Ginecobstetricia 'Luis Castelazo Ayala', Instituto Mexicano del Seguro Social, Apartado Postal 99-065, \\ Unidad Independencia, Mexico D.F. CP 10101, Mexico \\ ${ }^{2}$ Departamento de Fisicoquímica, Facultad de Química, Universidad Nacional Autónoma de México, C.U., México D.F. CP04510, Mexico \\ ${ }^{3}$ Departamento de Física Aplicada, Facultad de Física, Universidad de Santiago de Compostela, E-15782 Santiago de Compostela, Spain \\ ${ }^{4}$ Oregon National Primate Research Center, Beaverton, 97006 Oregon, USA \\ (Correspondence should be addressed to A Ulloa-Aguirre; Email: aulloaa@servidor.unam.mx) \\ *(E Jardón-Valadez and A Aguilar-Rojas contributed equally to this work)
}

\begin{abstract}
In the present study, we analyzed the role of Lys191 on function, structure, and dynamic behavior of the human GnRH receptor (hGnRHR) and the formation of the Cys14-Cys200 bridge, which is essential for receptor trafficking to the plasma membrane. Several mutants were studied; mutants lacked either the Cys14-Cys200 bridge, Lys191 or both. The markedly reduced expression and function of a Cys14Ser mutant lacking the 14-200 bridge, was nearly restored to wild-type/ $\Delta$ Lys191 levels upon deletion of Lys191. Lys191 removal resulted in changes in
\end{abstract}

the dynamic behavior of the mutants as disclosed by molecular dynamics simulations: the distance between the sulfur- (or oxygen-) sulfur groups of Cys (or Ser) 14 and Cys 200 was shorter and more constant, and the conformation of the $\mathrm{NH}_{2}$-terminus and the exoloop 2 exhibited fewer fluctuations than when Lys191 was present. These data provide novel information on the role of Lys191 in defining an optimal configuration for the hGnRHR intracellular trafficking and function.

Journal of Endocrinology (2009) 201, 297-307

\section{Introduction}

The mammalian GnRH receptor (GnRHR) type I (hereafter referred as GnRHR) belongs to the superfamily of G-protein coupled receptors (GPCRs), specifically, to the family related to the rhodopsin- and $\beta_{2}$-adrenergic-like receptors (Family A; Ulloa-Aguirre \& Conn 1998, Millar et al. 2004). The GnRHR is located in the pituitary gonadotroph and is bathed by the circulation of the hypothalamic-pituitary portal system which transfers pulsatile signals of the hypothalamic decapeptide, GnRH. The gonadotroph cell responds with a concomitant pulsatile release of the gonadotrophins, LH and FSH (Santen \& Bardin 1973, Knobil 1974). These enter the peripheral circulation and regulate gonadal steroidogenesis, along with gametogenesis.

The GnRHR is among the smallest members of the GPCR superfamily (328 amino acid residues in the human GnRHR (hGnRHR)); unlike other members of the rhodopsin/ $\beta$-adrenergic subfamily of GPCR, including the type II GnRHR (Millar 2003), the GnRHR exhibits several unique features such as the reciprocal change of the conserved Asp and Asn residues in transmembrane domains (TM) 2 and 7 (Awara et al. 1996), the replacement of Tyr with Ser in the highly conserved Asp-Arg-Tyr (DRY) motif located in the junction of the TM3 and the intracellular loop (IL) 2, and the lack of the carboxyl-terminal extension into the cytosol (McArdle et al. 1999, Millar 2003, Millar et al. 2004), whose presence is associated with differential physiological receptor regulation (Heding et al. 1998, Lin et al. 1998). Another important feature of the GnRHR is the amino acid residue at position 191 in the extracellular loop (EL) 2, which is frequently Glu or Gly in many mammals, but is replaced with Lys in primates (Janovick et al. 2006, Ulloa-Aguirre et al. 2006); in rat and mice GnRHR (327 amino acid residues) this amino acid is absent, conferring the GnRHR increased cell surface membrane expression for agonist (Arora et al. 1999). Knowledge of the structural requirements that govern GnRHR intracellular trafficking and cell surface membrane expression is of prime importance considering that this receptor is presently a therapeutic target for a number of pathological conditions such as prostate cancer, uterine fibroids, endometriosis, and precocious puberty.

The amino acid sequence of the hGnRHR predicts formation of two disulfide bridges at extracellular regions, one connecting the first and second ELs (Cys114-Cys196) and the second connecting the amino terminal extension 
with the EL2 (Cys14-Cys200; Millar et al. 2004). The first bridge that involves two highly conserved cysteine residues is a structural feature present in many GPCRs and it is associated with the fundamental stability of the seven transmembrane structure (Ulloa-Aguirre \& Conn 1998). The functional significance of the Cys14-Cys200 bridge differs depending on the receptor species (Knollman et al. 2005). In the rat, this bridge is not essential for optimal function of the GnRHR as replacement of the Cys residues at either end of the bridge does not affect plasma membrane expression and agonist-stimulated intracellular signaling (Cook \& Eidne 1997, Janovick et al. 2002), whereas in the mouse GnRHR, breakage of this bridge results in $\sim 50 \%$ decrease in receptor function (Knollman et al. 2005). In the human receptor, formation of this bridge is an absolute requirement for efficient routing and plasma membrane expression of the receptor as bridge-breaking mutants Cys200Tyr (a naturally occurring mutation in humans; Beranova et al. 2001), Cys14Ala and Cys200Ala (Janovick et al. 2002) exhibit either none or marginal activity; further, exposure to these mutants to pharmacological chaperones (i.e., small molecules that serve as molecular scaffolding to promote correct folding of otherwise misfolded mutant proteins), normalize receptor function indicating that the absence of the bridge resulted in a misfolded protein (Leanos-Miranda et al. 2002, Knollman et al. 2005).

The structural determinants that lead to the requirement of the Cys14-Cys200 bridge in folding and plasma membrane expression of the hGnRHR have been defined by mutagenesis experiments (Janovick et al. 2006, 2007). These studies revealed that residues located in the $\mathrm{NH}_{2}{ }^{-}$ terminus and in the EL2 as well as sequences flanking this loop (i.e., within TMs 4 and 5) and those that abut on that area (ELs 1 and 3), presumably control the destabilizing role of Lys191 on the formation of the Cys14-Cys200 bridge. In fact, removal of Lys191 from misfolded hGnRHR mutants (including Cys14Ala and Cys200Tyr mutants) led to partial or complete functional recovery of the altered receptors indicating that the association Cys14-Cys200 may be potentially disrupted or diminished by the presence of Lys191, which is present in primate GnRHRs and absent in mice and rat receptors (Leanos-Miranda et al. 2002, Janovick et al. 2006).

In the present study, we applied a combined strategy (mutagenesis and functional studies as well as computational modeling and molecular dynamics (MD) simulations) to analyze the role of Lys191 on the functional, structural, and dynamic behavior of the hGnRHR. A model of the wildtype (Wt) hGnRHR in explicit dipalmitoyl phosphatidyl choline bilayers, recently developed in our laboratory and further refined by intensive MD simulations (Jardon-Valadez et al. 2008), was employed as reference structure for the mutations and structural modifications. Both experimental and theoretical results provide new insights at the atomic level into this issue, and indicate that the absence of Lys191 favors the interactions between the EL2 and the $\mathrm{NH}_{2}$-teminus, the formation of the Cys14-Cys200 disulfide bridge, and the acquisition of a hGnRHR configuration compatible with high-plasma membrane expression and function, even in the absence of the Cys14-Cys200 bridge.

\section{Materials and Methods}

\section{Construction of $h \mathrm{GnRHR}$ mutants}

A hGnRHR lacking the apparent disulfide bridge at positions 14 and 200 was constructed by replacing Cys at position 14 of the Wt receptor with Ser as previously reported (Davidson et al. 1997). Construction of the hGnRHR Cys14Ser mutant was performed employing the full-length Wt hGnRHR cloned into pcDNA3.1 at KpnI and XbaI restriction enzyme sites. Site-directed mutagenesis was performed using the Quick Change site-directed mutagenesis kit (Stratagene, La Jolla, CA, USA), following the manufacturer instructions. Mutagenic oligonucleotides (Life Technologies; forward: 5'-GAACAGAATCAAAATCACTCTTCAGCCATCAACAACAGC-3'; reverse: 5'-GCTGTTGTTGATGGCTGAAGAGTGATTTTGATTCTGTTC-3') were designed according to the cDNA sequence reported for the hGnRHR (GenBank accession no. L07949; Chi et al. 1993). The hGnRHR Cys14Ser/DLys191 mutant was constructed by the above described mutagenesis procedure employing a $\mathrm{Wt}$ receptor sequence (cloned in pcDNA3.1) lacking lysine at position 191 (hGnRHR/DLys191; Maya-Nunez et al. 2002) as template. For transfection, large-scale plasmid DNAs were prepared using an Endofree maxiprep kit (Qiagen). The identity of all constructs was verified by automated sequencing employing the BigDye Terminator Cycle Sequence kit (Applied Biosystems, Foster City, CA, USA).

\section{Transient transfection of COS-7 cells}

Wt and mutant hGnRHRs were transiently expressed in COS-7 cells as described (Maya-Nunez et al. 2002). Fifty thousand or 1000 cells per well were plated in 48-well plates (for assessing inositol phosphate (IP) production) or 24-well plates (for binding experiments; Costar, Cambridge, MA, USA), respectively, and $20 \mathrm{~h}$ later the cells were transfected with $0 \cdot 050$ or $0.2 \mu \mathrm{g}$ (for IP production or binding studies respectively) hGnRHR DNA constructs per well, using liposome-mediated endocytosis, as described (Leanos-Miranda et al. 2005). After transfection, the cells were washed twice with DMEM/0 $1 \%$ BSA/gentamicin and preloaded with $4 \mu \mathrm{Ci} / \mathrm{ml}\left[{ }^{3} \mathrm{H}\right]$-myoinositol (for IP assays) or DMEM (for binding studies) as described below. IP production was measured after exposure of the cells to the GnRH agonist, Buserelin (Sigma) for $2 \mathrm{~h}$.

\section{Measurement of IP production}

Quantification of IP production by Dowex anion exchange chromatography and liquid scintillation spectroscopy was performed as described previously (Huckle \& Conn 1987). 


\section{Receptor binding assay}

COS-7 cells were transiently transfected as described above. Twenty hours after the start of transfection, the cells were washed twice with warm DMEM/0 $1 \%$ BSA/10 mM HEPES and cultured in DMEM for $18 \mathrm{~h}$ before addition of $\left[{ }^{125} \mathrm{I}\right]-$ Buserelin (specific activity $700 \mu \mathrm{Ci} / \mu \mathrm{g}$ ). Cells were incubated at room temperature for $90 \mathrm{~min}$ in the presence or absence of excess $(10 \mu \mathrm{M})$ unlabelled ligand (Buserelin; Sigma) plus $\left[{ }^{125} \mathrm{I}\right]$-Buserelin. Thereafter, the medium was removed, the plates containing the cells were placed on ice, washed twice with ice-cold PBS, and then the cells were solubilized by the addition of $0 \cdot 2 \mathrm{M} \mathrm{NaOH} / 0 \cdot 1 \% \mathrm{SDS}$. Aliquots of samples were then transferred to glass tubes and counted in a $\gamma$-counter (Packard Instruments, Downers Grove, IL, USA). Specific binding was calculated by subtracting non-specific binding (binding measured in the presence of $10 \mu \mathrm{M}$ Buserelin) from total binding (no GnRH agonist added).

For the radioreceptor assay, COS-7 cells were transfected as described above and incubated at room temperature for $90 \mathrm{~min}$ in the presence or absence of excess Buserelin plus $\left[{ }^{125} \mathrm{I}\right]$-Buserelin or $\left[{ }^{125} \mathrm{I}\right]$-Buserelin plus increasing concentrations $\left(10^{-11}\right.$ to $\left.10^{-6}\right)$ of the unlabelled ligand. Thereafter, the medium was removed and the plates containing the cells were placed on ice and washed twice with ice-cold PBS. The cells were then solubilized by the addition of $0.2 \mathrm{M}$ $\mathrm{NaOH} / 0 \cdot 1 \%$ SDS. Aliquots of samples were then transferred to glass tubes and counted in a $\gamma$-counter.

\section{Mutations and structural modifications on the Wt $h \mathrm{GnRHR}$ for computational modeling and MD simulations}

Five mutations/structural modifications were introduced on a refined hGnRHR model (Jardon-Valadez et al. 2008). Mutant hGnRHR Cys14\|200Cys was identical to the original model but with the Cys14-Cys200 disulfide bridge disrupted by reducing both Cys residues. Lysine 191 was deleted in the Wt hGnRHR structure to yield the hGnRHR/DLys191 structure. The two previous structural modifications were simultaneously applied to generate the mutant hGnRHR Cys14||200Cys/DLys191. To propose structure-functional relationships with the results obtained from the mutagenesis and biochemical experiments, GnRHR Cys14Ser and hGnRHR Cys14Ser/ $\Delta$ Lys191 mutant computational models were also generated and simulated. Residue substitutions were performed by keeping the backbone atom coordinates of the original structure and replacing the side chains. Rupture of disulfide bridges required the elimination of the corresponding bond restraint from the potential function and the addition of the reducing hydrogen atom in both Cys residues. Deletion of Lys191 was directly performed on the coordinates file of the $\mathrm{Wt}$ receptor model, with the peptide bond between Thr190 and Val192 being imposed. In all cases, the steepest descent minimization was performed to prevent unfavorable contacts and highly unstable interactions. Water molecules and lipids present in the original model were preserved in such a manner that only the local environment of the modified EL2 was perturbed. After minimization, a short molecular dynamics relaxation was performed and thereafter three different 35-ns-long MD simulations at $310 \mathrm{~K}$ and constant volume were performed for each system. The temperature of the simulations was chosen to reproduce physiological conditions of the protein. Three MD simulations of the refined Wt hGnRHR model (JardonValadez et al. 2008) were performed and used as control runs.

\section{Simulation parameters}

All systems consisted of 15449 water molecules, 192 lipid molecules, the mutant receptor structure, and 15 or 14 chlorine ions, depending on the presence or absence of Lys191. Periodic boundary conditions were imposed for a simulation box of $7.6 \times 7.6 \times 13 \mathrm{~nm}^{3}$. A total of $18 \mathrm{MD}$ simulations were performed using the GROMACS package (version 3.3.1; Berendsen et al. 1995, Lindahl et al. 2001, Van Der Spoel et al. 2005). The extended simple point charge (SPC/E) water model (Berendsen et al. 1987) was used while the $53 \mathrm{a} 6$ parameterization of the GROMOS96 force field (Oostenbrink et al. 2004) was employed for the protein and the lipid molecules. Water, protein, lipids, and ions were separately coupled to an external temperature bath (using a coupling constant of $0.1 \mathrm{ps}$ ) by means of a Berendsen thermostat (Berendsen et al. 1984). The long-range interactions were calculated using the efficient Particle Mesh Ewald (Darden et al. 1993, Essman et al. 1995) with a real space cut-off of $0.9 \mathrm{~nm}$, a $0.12 \mathrm{~nm}$ space grid, and a fourth order B-spline interpolation scheme. The equations of motion were integrated using the leap-frog method (Hockney \& Eastwood 1988) with a 2 fs time step. The bond lengths and $\mathrm{H}-\mathrm{O}-\mathrm{H}$ angle in water were constrained using the SETTLE algorithm (Miyamoto \& Kollman 1992), while the LINCS algorithm (Hess et al. 1997) was used to constrain bond lengths in the receptor and lipid molecules. All atom coordinates were stored every $10 \mathrm{ps}$. The initial velocities of the atoms were randomly assigned to produce a Maxwell distribution corresponding to the temperature at which the simulation will be performed.

\section{Analysis of the trajectories}

Root mean square positional deviations (RMSD) of the whole protein backbone, the backbone of the transmembranal domains, and the backbone atoms of the ELs together with those of the $\mathrm{NH}_{2}$-terminus were calculated throughout the trajectories. Additionally, in order to compare the time evolution of the mutants' trajectories with those of the $\mathrm{Wt}$ receptor, RMSD matrices for the $\alpha$-carbons were calculated. These matrices provide a global comparison of every structure reached along a particular trajectory of a mutant with those sampled for the $\mathrm{Wt}$ receptor in a different trajectory. RMS positional fluctuations (RMSF) per residue were also calculated over the last 15 ns of the trajectory. The S-S (or O-S in Cys14Ser mutants) distances between the sulfur 
atom of Cys14 (or the oxygen atom of Ser14) and that of Cys200 were determined as a function of time. The blocking average method (Flyvbjerg \& Petersen 1989) was used to calculate the S.D. for the S-S distance. Hydrogen bonds between extracellular amino acid residues were also calculated for the trajectories involving the Cys14Ser mutation using a donor-acceptor atom cutoff distance of $3.5 \AA$ and a donorhydrogen-acceptor angle of $<30^{\circ}$. All analyses were performed using tools from the GROMACS 3.3.1 package (Berendsen et al. 1995, Lindahl et al. 2001, Van Der Spoel et al. 2005), the viewers RASMOL 2.7 (Sayle \& Milnerwhite 1995), VMD 1.8.2 (Humphrey et al. 1996), Pymol 0.99 (De Lano 2002), and several computer programs and scripts specifically developed for this purpose.

\section{Statistical analysis}

Data from the biochemical studies were analyzed with oneway ANOVA and then with the Walker-Duncan adaptive procedure. Binding parameters were calculated from the doseresponse displacement curves using the software GraphPad Prism 4.0 (GraphPad Software Inc., La Jolla, CA, USA).

\section{Results}

\section{Functional studies}

As shown in Fig. 1A, all hGnRHRs expressed in COS-7 cells specifically bound ${ }^{125} \mathrm{I}$-Buserelin. In the absence of the
Cys14-Cys200 disulfide bridge (hGnRHR Cys14Ser mutant) maximally bound radiolabelled GnRH analog was considerably reduced (at levels $17 \pm 2 \%$ from that exhibited by the $\mathrm{Wt}$ receptor). The binding capability of $\mathrm{Wt}$ and mutant hGnRHRs to which Lys191 was removed, markedly increased as compared with their corresponding counterparts bearing this amino acid. Maximal binding of radiolabelled Buserelin to the Cys14Ser/DLys191 mutant, however, remained below (at levels $67 \pm 8 \%$ ) that shown by the $\mathrm{Wt}$ hGnRHR. The reduced maximal ${ }^{125}$ I-Buserelin binding of the hGnRHR Cys14Ser/DLys191 mutant was apparently due to a decreased relative binding affinity of agonist for this altered receptor (Fig. 1B and Table 1), since the number of functional mutant Cys14Ser/ $\Delta$ Lys191 receptors at the cell surface increased to levels between those of $\mathrm{Wt}$ receptor bearing and lacking Lys191.

Despite the markedly reduced analog binding capacity of the hGnRHR lacking the Cys14-Cys200 disulfide bridge this modification did not abolish completely agoniststimulated activation of the receptor (Fig. 1C and Table 1). Replacement of Cys with Ser at position 14, resulted in $\sim 50 \%$ reduction in maximal IP production in response to saturation concentration of the metabolically stable agonist Buserelin. By contrast, deletion of Lys191 from the Wt and the Cys14Ser mutant receptor provoked a shift of the corresponding IP dose-response curves to the left, yielding comparable $\mathrm{ED}_{50}$ values $(17 \cdot 1 \pm 4$ and $19 \cdot 1 \pm 3 \mathrm{pM}$ for the Wt and mutant hGnRHRs lacking Lys191 respectively) and maximal agonist-stimulated IP production (Table 1).
A

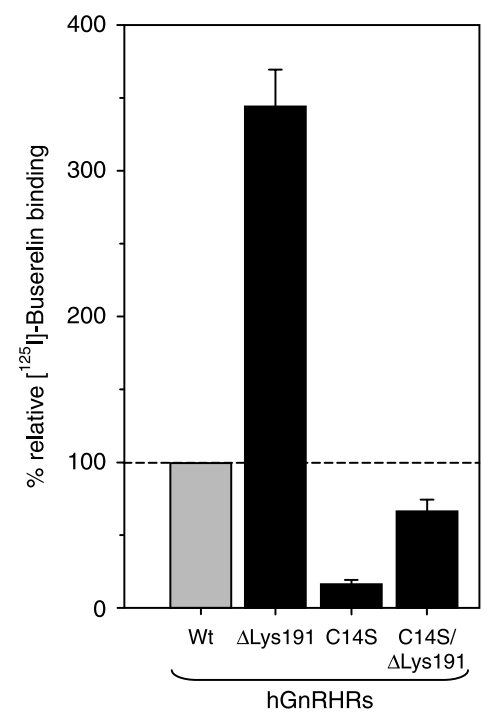

B

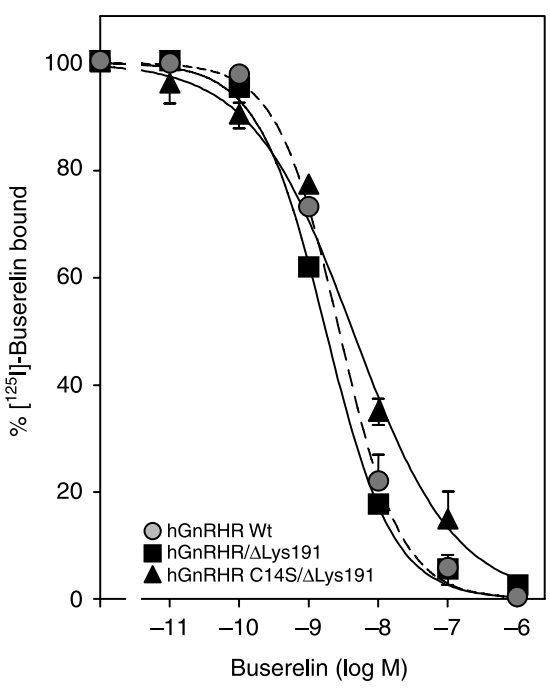

C

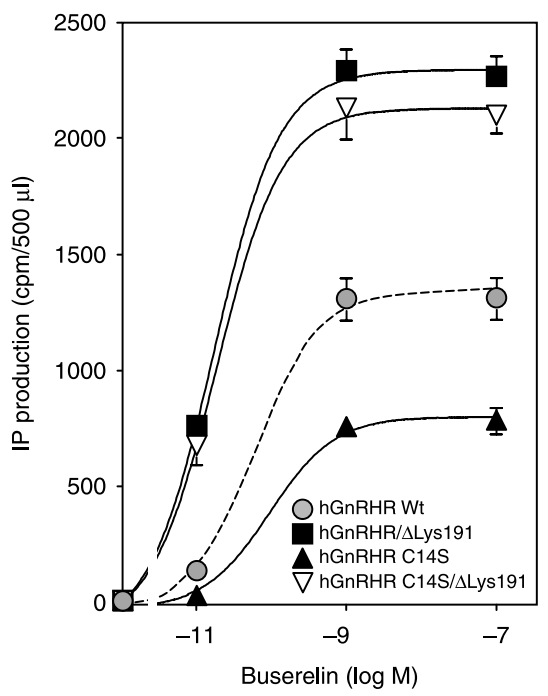

Figure 1 (A) Specific labeled agonist binding to culture COS-7 cells transiently expressing the hGnRHR/DLys191, Cys14Ser, and

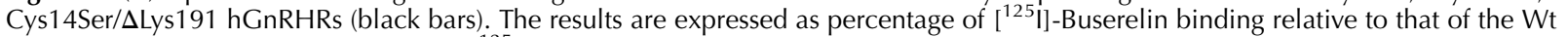
hGnRHR (grey bar). (B) Displacement of [ $\left.{ }^{125} \mathrm{I}\right]-$ Buserelin by increasing concentrations of unlabelled GnRH agonist in COS-7 cells transfected with the Wt and modified hGnRHRs. (C) Inositol phosphate (IP) dose-response curves for Buserelin in cultured COS-7 cells transiently

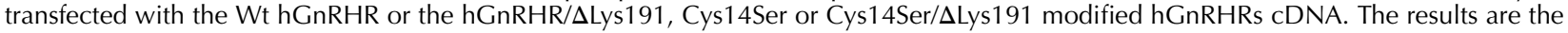
means \pm s.D. of three independent experiments each carried out in triplicate incubations. 
Table 1 Binding parameters, maximal Buserelin-stimulated inositol phosphate (IP) production, and $\mathrm{ED}_{50}$ of the wild-type (Wt) and mutant hGnRHRs (means \pm s.E.M. from three independent experiments)

\begin{tabular}{|c|c|c|c|c|}
\hline & GnRH IC 50 (nM) & $\begin{array}{l}\text { Cell surface receptor } \\
\left.\text { (fmol } 110^{5} \text { cells }\right)\end{array}$ & $\begin{array}{l}\text { Max IP production } \\
\quad(\% \text { GnRHR Wt })\end{array}$ & $\mathbf{E D}_{50}(\mathrm{pM})$ \\
\hline \multicolumn{5}{|l|}{ hGnRHR } \\
\hline hGnRHR Wt & $2 \cdot 4 \pm 0 \cdot 2^{\mathrm{a}}$ & $5 \cdot 5 \pm 0 \cdot 7^{\mathrm{a}}$ & $100 \pm 4^{a}$ & $58 \cdot 9 \pm 5 \cdot 8^{\mathrm{a}}$ \\
\hline hGnRHR/DLys191 & $1 \cdot 5 \pm 0 \cdot 1^{b}$ & $15 \cdot 6 \pm 1 \cdot 0^{\mathrm{b}}$ & $174 \pm 8^{b}$ & $17 \cdot 1 \pm 0 \cdot 5^{b}$ \\
\hline hGnRHR Cys14Ser & - & - & $60 \pm 3^{c}$ & $82 \cdot 8 \pm 3 \cdot 1^{\mathrm{c}}$ \\
\hline hGnRHR Cys14Ser/DLys191 & $5 \cdot 3 \pm 0 \cdot 7^{\mathrm{c}}$ & $9 \cdot 6 \pm 0 \cdot 9^{b}$ & $162 \pm 15^{b}$ & $19 \cdot 1 \pm 2 \cdot 3^{b}$ \\
\hline
\end{tabular}

Values with different superscript letters within each column are statistically $(P<0 \cdot 05)$ different.

In silico analysis of Wt and mutant $h G n R H R s$ structure

To assess the role of lysine at position 191 on receptor conformation, three independent trajectories for each mutant generated were monitored by all-atom MD simulations. In all runs, the overall protein structure was well preserved as disclosed by the convergence of the backbone RMSD shown in Figure S1 (see Supplementary data in the online version of the Journal of Endocrinology at http://joe.endocrinologyjournals.org/content/vol201/issue2/). RMSD values for the three replicas and the contribution for the helices backbone are also included in the figure. Except for a run in the hGnRHR Cys14\|200Cys mutant, all RMSD values were lower than $3 \cdot 0 \AA$ throughout the corresponding trajectories. Likewise, the RMSD values for the helices backbone was close to $2 \cdot 0 \AA$ in all cases. Similar deviations from those configurations sampled in an extended trajectory of the hGnRHR model were detected in all mutant structures (Figure S2, see Supplementary data in the online version of the Journal of Endocrinology at http://joe.endocrinologyjournals.org/content/vol201/issue2/). Removal of the Cys14-Cys200 disulfide bridge by reducing both Cys residues (hGnRHR Cys14\|200Cys) or replacing Cys14 by Ser (mutant hGnRHR Cys14Ser) as well as elimination of lysine at position 191 (mutant hGnRHR/DLys191) from the Wt receptor resulted in evident conformational changes on the simulated receptor structures. The double mutations involving deletion of Lys191 (mutants hGnRHR

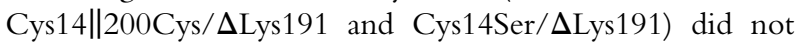
present major conformational changes as revealed by their corresponding RMSD values which were comparable with the contributions of the single mutations. In fact, the configuration of hGnRHR Cys14\|200Cys/DLys191 would seem to be more similar to the Wt structure than the Cys14\|200Cys receptor (which includes Lys191; Figure S2). As mentioned above, the structural stability of the helice domains suggested that major conformational changes were expected to occur in the extracellular domains $\left(\mathrm{NH}_{2}-\right.$ terminus and ELs). The RMSDs for the extracellular domains (backbone atoms) and the second EL from one representative run are separately shown in Fig. 2; equivalent plots for the other two runs are included in Figure S3 (see Supplementary data in the online version of the Journal of Endocrinology at http://joe.endocrinology-journals.org/content/vol201/ issue2/). Similar time-related profiles were observed for each pair of RMSD, indicating that this analysis was not able to clearly distinguish between different mutants. These plots, together with the RMSD values displayed in Figure S1, also show that the main contribution to the conformational changes of the extracellular domain came from the EL2 and its environment.
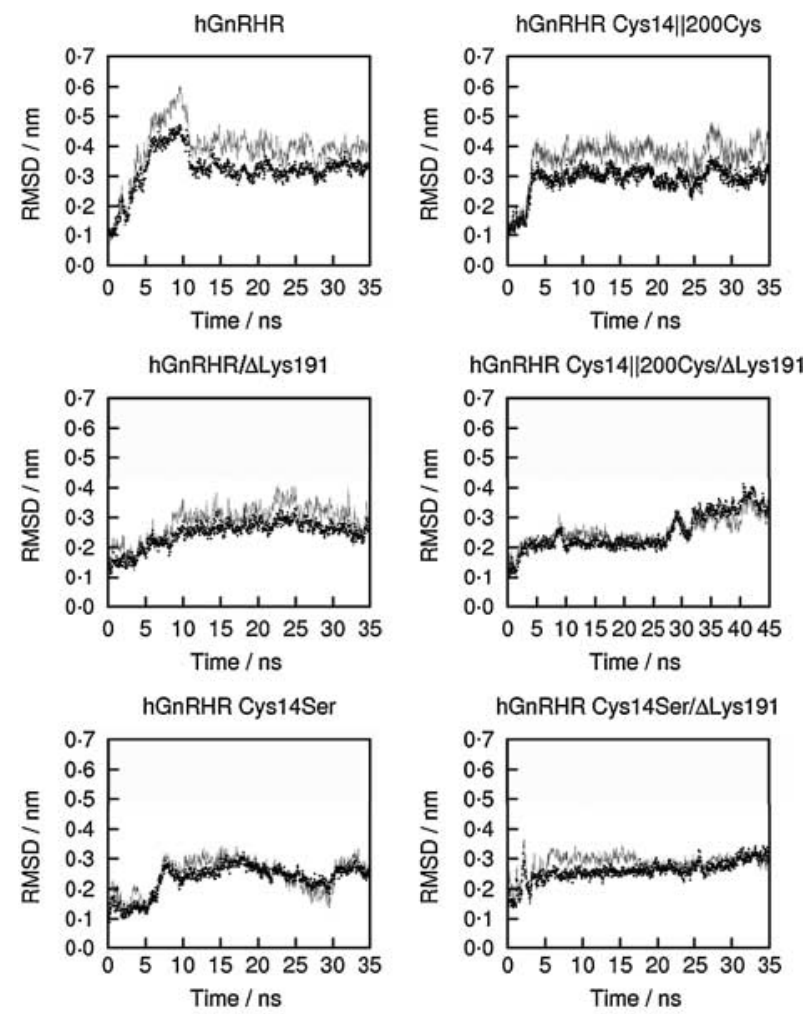

Figure 2 Root mean square positional deviations (RMSD) for the backbone of the extracellular domains $\left(\mathrm{NH}_{2}\right.$-terminus and $\left.\mathrm{ELs}\right)$ as a function of time (gray solid lines), and for the backbone of the second extracellular loop (EL2; black dots). Extracellular conformational changes are mostly determined by the EL2 segment. 
Figure S4 (see Supplementary data in the online version of the Journal of Endocrinology at http://joe.endocrinologyjournals.org/content/vol201/issue2/) shows the RMSFs per residue for the $\mathrm{Wt}$ and mutant structures. Residues in the TMs 1-7 were relatively static, while major mobilities were observed in the intra- and ELs, as usually found in other GPCRs (Colson et al. 1998). Lysine 191 in the Wt and hGnRHR Cys14\|200Cys structures (or Val192 in

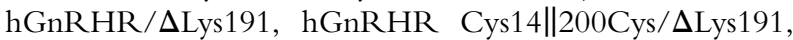
and hGnRHR Cys14Ser/ALys191) is located in the EL2, which is a highly-dynamic domain. In general, maxima and minima RMSF values appeared in the same receptor domains for all mutants and replicas, and although no clear correlations with the presence or absence of Lys191 were detected by this type of analysis, larger fluctuations were observed in those mutants including Lys191 (Figure S4). Nevertheless, differences in Cys14-Cys200 (or Ser14-Cys200) distances were noticed between the hGnRHR Cys14\|200Cys and hGnRHR Cys14||200Cys/DLys191 (or hGnRHR Cys14Ser and hGnRHR Cys14Ser/DLys191) structures. Figure 3 and Table 2 show the distance between sulfur atoms of Cys 14 and Cys200 for the hGnRHR Cys14\|200Cys and hGnRHR Cys14\|200Cys/DLys191 structures as well as that between the oxygen atom of Ser14 and Cys200 for the hGnRHR Cys14Ser and hGnRHR Cys14Ser/DLys191 structures as a function of time and for the three replicas of each system. Specifically, in structure hGnRHR Cys14||200Cys, the S-S distance increased from the initial bond distance to an average of $9 \cdot 85 \pm 0 \cdot 45 \AA$ in the second run and to $6 \cdot 22 \pm 0 \cdot 11 \AA$ in the third run. By contrast, in hGnRHR Cys14\|200Cys/ $\Delta$ Lys191 (lacking Lys191) the S-S distance was shorter and more constant in the three replicas even in the absence of the 14-200 disulfide bridge. In the hGnRHR Cys14Ser structure (bearing Lys191), the $\mathrm{O}-\mathrm{S}$ distance increased up to $7 \cdot 74 \pm 0 \cdot 23 \AA$ in the first run and was clearly larger in the other two runs than in hGnRHR Cys14\|200Cys/ $\Delta$ Lys191. For the mutant hGnRHR Cys14Ser/DLys191, the O-S distance average was $3 \cdot 75 \pm 0.08 \AA$ and $4 \cdot 45 \pm 0 \cdot 10 \AA$ for the first and second run respectively. Thus, a trend towards more constant and shorter distances between residues at positions 14 and 200 (at the $\mathrm{NH}_{2}$-terminus and the EL2 respectively) was present in those structures lacking Lys191. The first run of hGnRHR Cys14\|200Cys and the third run in hGnRHR Cys14Ser/ $\Delta$ Lys191 may be considered as borderlines for this trend. Computational limitations did not allow generation of significantly longer trajectories that may clarify whether the corresponding conformations were kinetically trapped. The distances between the residues 14 and 200 of selected final configurations are compared with Wt structure in Fig. 3.

Finally, H-bond networks on hGnRHR Cys14Ser and hGnRHR Cys14Ser/DLys191 mutants were analyzed. H-bond pathways involving Ser14, Ser201 (Ser200 when Lys was absent), Ser301 (Ser300), Asp302 (Asp301), and several water molecules around these residues were clearly observed in both receptor structures. These interactions allowed preservation of a serine-rich microdomain even in the absence of the Cys14-Cys200 bridge (Fig. 4A). Interestingly, the formation of those $\mathrm{H}$-bond networks involved less water molecules in the absence of Lys191 (Fig. 4B).

\section{Discussion}

As with other GPCRs, a large percentage of the Wt hGnRHR is normally retained in the endoplasmic reticulum and never reaches the cell surface plasma membrane (Ulloa-Aguirre et al. 2004, Conn et al. 2007). This limited plasma membrane expression of the Wt hGnRH contrasts with that shown by the Wt rat GnRHR, whose cell surface membrane expression exceeds, by more than twofold, that presented by its human counterpart (Janovick et al. 2003b, 2006). Among the structural differences between these GnRHRs is the presence of Lys191 in the EL2 of the human receptor, which is frequently glutamic acid or glycine in other non-primate mammalian species (Arora et al. 1999, Janovick et al. 2006, Ulloa-Aguirre et al. 2006). Deletion of Lys191 from the hGnRHR or exposure to pharmacological chaperones, markedly increase plasma membrane expression of the Wt receptor to levels comparable with those of the rat GnRHR (Maya-Nunez et al. 2002, Janovick et al. 2003a,b, 2006), indicating that the presence of Lys191 limits plasma membrane expression of the human receptor presumably by destabilizing the protein structure (Arora et al. 1999). The presence and location of Lys191 in the hGnRHR is of particular interest, since the EL2 is one of the receptor regions showing the largest fluctuations in mobility as indicated by molecular dynamics simulation studies (Jardon-Valadez et al. 2008; present study). Although the entire EL2 consists of 27 amino acid residues the presence of two disulfide bonds divides this loop into three distinct segments separated by Cys196 and Cys200. The first segment has five hydrophobic and six polar, non-charged residues as well as Lys191, which exhibits the highest dynamic fluctuations within the EL2 of the receptor (Jardon-Valadez et al. 2008).

Mutational and biochemical studies have shown that Lys191 in the hGnRHR destabilizes the association between Cys14 and Cys200, which forms a disulfide bridge that is required for optimal routing of this particular receptor to the cell surface membrane (Janovick et al. 2006, Ulloa-Aguirre et al. 2006, Conn et al. 2007). Apparently, the effect of Lys191 is not wholly an effect of charge because replacement by Ala, Glu, or Gln is also associated with inefficient cell surface membrane expression of the Wt hGnRHR compared with the hGnRHR/DLys191 variant (Janovick et al. 2006). In fact, replacement of Cys 14 or Cys 200 with Ala, resulted in almost complete loss of IP production, which was restored to nearly Wt levels by pharmacological chaperones or by deleting Lys191 (Janovick et al. 2006), suggesting that the absence of this bridge in the presence of Lys191 yielded a misfolded protein. In the present study, a hGnRHR lacking the apparent disulfide bridge between residues 14 and 200 was constructed by replacing Cys at position 14 of the $\mathrm{Wt}$ 


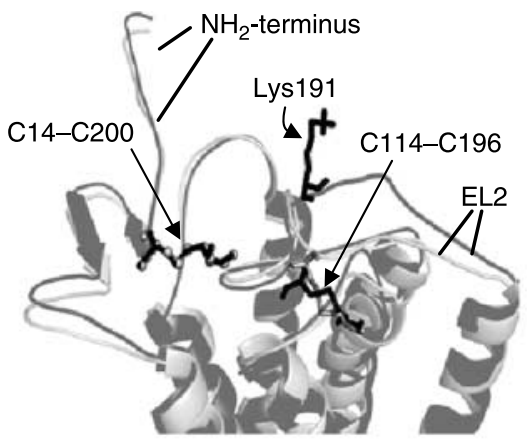

hGnRHR/ALys 191
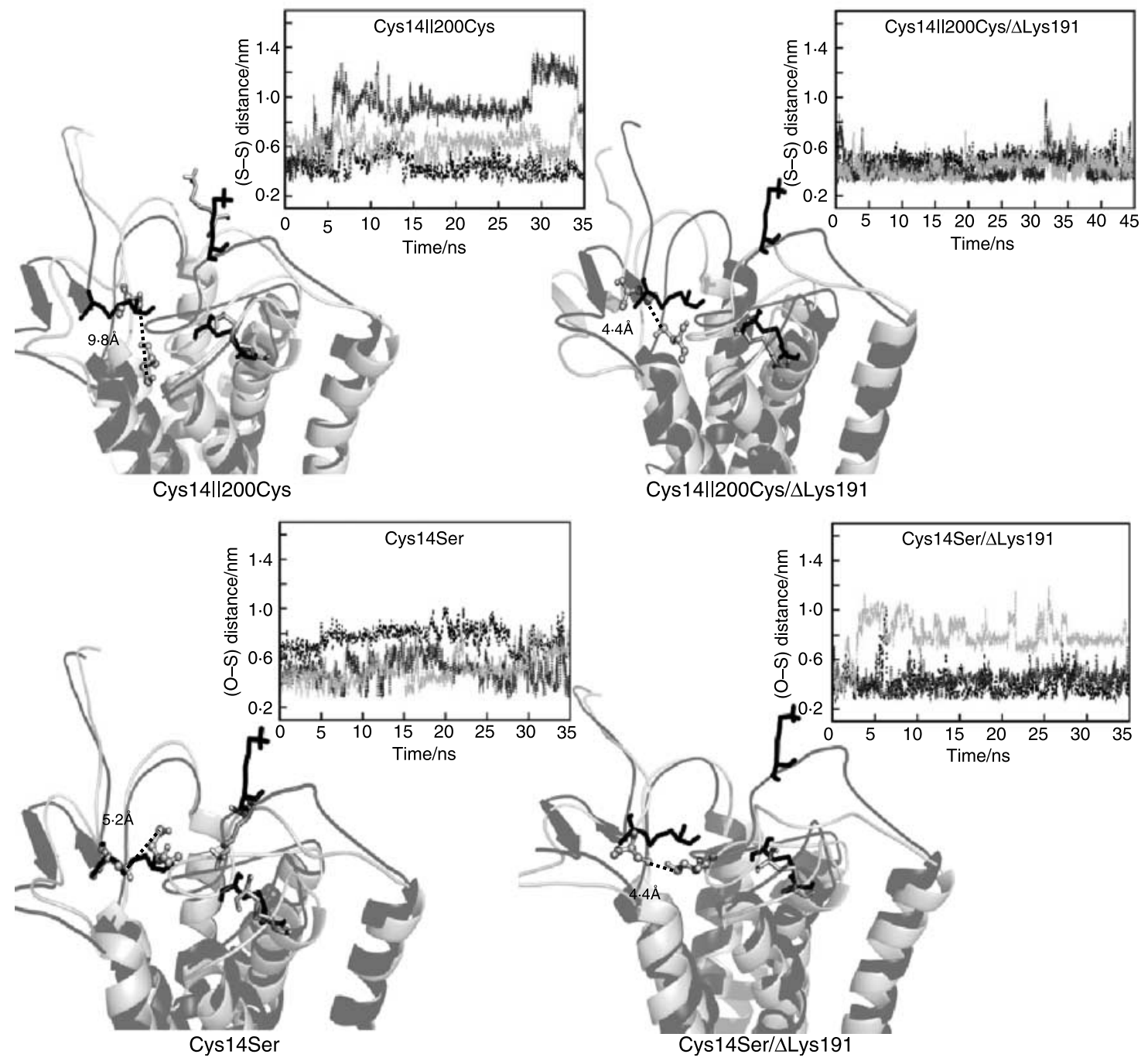

Figure 3 Superposition of the Wt hGnRHR conformation (dark structures) and the final mutant conformations (light structures) in simulation R2. Lysine 191 and the Cys14-Cys200 and Cys114-Cys196 disulfide bridges of the Wt hGnRHR are highlighted in black. Residues C14/S14 and C200 are depicted in balls and sticks. S-S and S-O distances between residues in positions 14 and 200 are also indicated (dotted lines). Insets: distances (in $\mathrm{nm}$ ) between sulfur and oxygen-sulfur atoms as a function of time for all replicas. Gray scale tones identify the individual simulation runs. Larger average distances and S.D.s are found in mutants bearing Lys191. 
Table 2 Average distances between sulfur atoms of Cys14-Cys200 or oxygen and sulfur of Ser14-Cys200 for the last 20 ns of each trajectory

\begin{tabular}{|c|c|c|c|c|}
\hline & & hGnRH & ant & \\
\hline & $\begin{array}{l}\text { Cys } 14 \| 200 \text { Cys } \\
\text { S-S distance (A) }\end{array}$ & $\begin{array}{c}\text { Cys } 14 \| 200 \text { Cys/ } / \text { Lys } 191 \\
\text { S-S distance }(\AA)\end{array}$ & $\begin{array}{c}\text { Cys14Ser } \\
\text { O-S distance }(\AA)\end{array}$ & $\begin{array}{l}\text { Cys14Ser/ALys } 191 \\
\text { O-S distance }(\AA)\end{array}$ \\
\hline Ru & & & & \\
\hline 1 & $4 \cdot 14 \pm 0 \cdot 08$ & $4 \cdot 74 \pm 0 \cdot 10$ & $7 \cdot 74 \pm 0 \cdot 23$ & $3 \cdot 75 \pm 0 \cdot 08$ \\
\hline 2 & $9 \cdot 85 \pm 0 \cdot 45$ & $4 \cdot 46 \pm 0 \cdot 21$ & $5 \cdot 24 \pm 0 \cdot 22$ & $4 \cdot 45 \pm 0 \cdot 10$ \\
\hline 3 & $6 \cdot 22 \pm 0 \cdot 11$ & $4 \cdot 58 \pm 0 \cdot 07$ & $5 \cdot 39 \pm 0 \cdot 34$ & $7 \cdot 89 \pm 0 \cdot 11$ \\
\hline
\end{tabular}

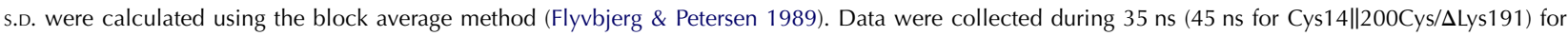
every $10 \mathrm{ps}$.

receptor with Ser. Despite binding of the GnRH agonist being reduced, the Cys14Ser mutant receptor displayed clearly detectable functional activity $(60 \%$ of that exhibited by the Wt receptor) but with a rightward shift in the dose-response curve for IP production. These findings are fully consistent with previous data (Davidson et al. 1997) and suggest that they more likely resulted from both decreased cell surface membrane expression and low-agonist binding provoked by configurational changes in the $\mathrm{NH}_{2}$-terminus and also probably in the EL2 (see below). Furthermore, removal of Lys191 from this particular Cys14-Cys200 bridgelacking mutant completely restored both plasma membrane expression and agonist-stimulated IP production to levels comparable with those exhibited by the Wt receptor lacking Lys191, despite an approximately twofold reduction in binding affinity of the agonist. The fact that removal of Lys191 from the Wt receptor only slightly altered the binding affinity of the receptor for GnRH agonist (Arora et al. 1999; present study) and that, in contrast to the Cys14Ser mutant, removal of Lys191 from the Cys14Ala mutant restored receptor function to levels comparable with those of the $\mathrm{Wt}$ receptor but well below those achieved by the hGnRHR/ $\Delta$ Lys191 (Janovick et al. 2006) strongly suggests that replacement of Cys14 with Ser resulted in gain-of-function of the altered hGnRHR, in which binding affinity was dissociated from receptor activation. In this scenario, removal of Lys191 from the Cys14Ser mutant not only allowed maximal cell surface membrane expression of the receptor but also evidenced the effect of the substitution on the configuration of the receptor and its impact in receptor activation. A similar gain-of-function in receptor activity has been previously detected in a triple mutant hGnRHR with particular substitutions at positions 4,7 , and 10 of the $\mathrm{NH}_{2}$ terminus (Janovick et al. 2006). In the case of the hGnRHR Cys14Ser mutant, our structural analysis suggests that the serine residue was involved in the formation of an extracellular H-bond network (Fig. 4) that, on the one hand, stabilized a particular receptor conformation but, on the other, disturbed binding of agonist due to interactions with Asp302 (Millar et al. 2004). This can not be achieved by the Cys14Ala or the Cys14Tyr mutations due to the hydrophobic character of alanine and tyrosine. Although the H-bonds-stabilized conformation could affect the orientation and/or mobility of particular TM helices (e.g., TM1 and/or TM7) such structural changes could not be detected in our limited time-scale MD simulations. Nevertheless, it is conceivable that changes in the configuration of the $\mathrm{NH}_{2}$-terminus may potentially influence the orientation of the adjacent TM1, and indirectly the intramolecular interactions between Asn53, Asn87 (in TM2), and Asp319 (in TM7) necessary for favoring the equilibrium between the active and inactive conformations of the receptor towards the inactive state (Millar et al. 2004). Changes in the conformation of the TM1 may also affect the interaction between Lys36 and Asn102 (Jardon-Valadez et al. 2008), the latter involved in GnRH binding (Davidson et al. 1996).

Previous mutagenesis studies have documented the functional impact of the differences between the rat GnRHR (no Lys191, high plasma membrane expression) and the hGnRHR (Lys191 present, inefficient plasma membrane expression) sequences and identified those amino acid residues that control the destabilizing influence of Lys191 on the formation of the Cys14-Cys200 disulfide bridge (Knollman et al. 2005, Janovick et al. 2006). In the present study, we performed MD simulations to analyze the effects of Lys191 on the three-dimensional structure and dynamic behavior of the hGnRHR. For these studies, we departed from a previously refined model of the Wt hGnRHR in an explicit lipid bilayer (Jardon-Valadez et al. 2008). All single mutations resulted in configurational changes on the extracellular domains of hGnRHR. Dynamic and structural analysis revealed that the presence of Lys191 provokes a tension between residues at positions 14 and 200, whereas its absence shortens and makes the distance between this pair of residues more stable. Moreover, the distance between Ser14 and Cys200 in the Cys14Ser mutant seemed relatively short and regular (even in the presence of Lys191), probably due to the participation of Ser14 in H-bonds network that may favor receptor stabilization in an active conformation. These results are in line with the assumption derived from mutagenesis studies ( Janovick et al. 2006, Ulloa-Aguirre et al. 2006, Conn et al. 2007) regarding the crucial role of Lys191 as well as residues in positions $112,208,300$, and 302 in determining the final conformation of the EL2 and its association with the 
A

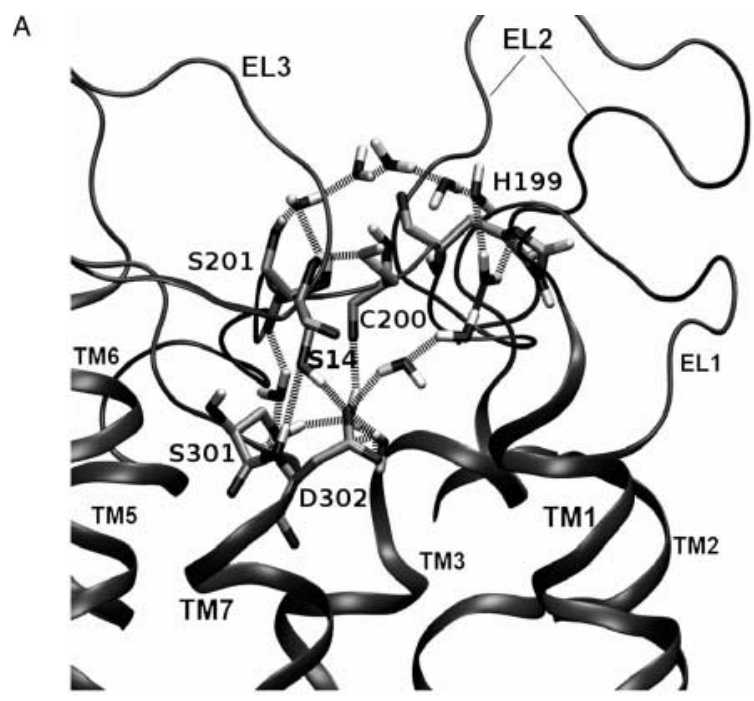

B

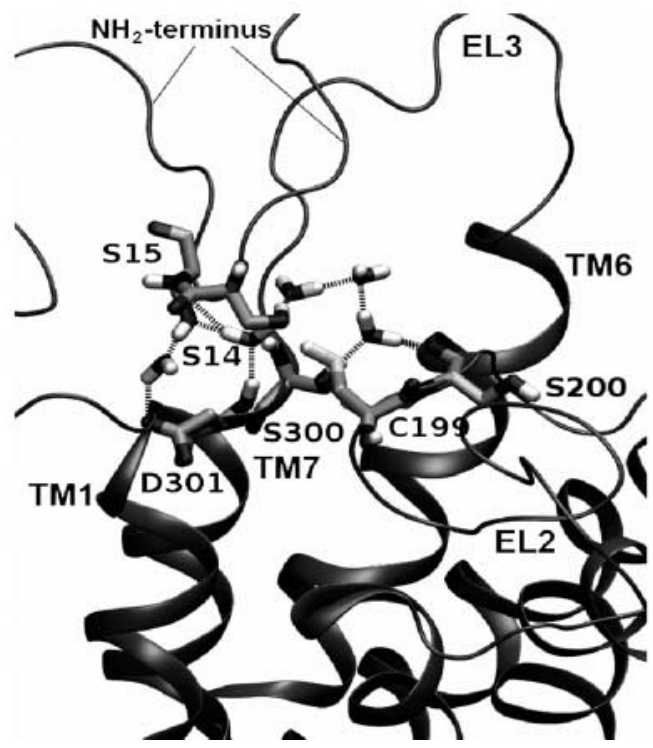

Figure 4 Snapshot showing hydrogen bond networks involving serine at position 14, water molecules, and residues of the ELs 2 and 3 in the presence (A) or absence (B) of Lys 191. TM, transmembrane domain; EL, extracellular loop.

$\mathrm{NH}_{2}$-terminal domain of the receptor. Apparently, the proximity between these two receptor domains represents a fundamental requisite for correct folding and intracellular trafficking of the GnRHR to the plasma membrane. In the Wt rat GnRHR, proximity between the $\mathrm{NH}_{2}$-terminus and the EL2 is ensured by specific amino acid residues located in several domains, including the TM4, EL2, and TM5, without the need for the formation of the Cys14-Cys199 bridge (Janovick et al. 2006), whereas in the Wt hGnRHR (which bears particular amino acids that co-evolved with and presumably control the destabilizing influence of Lys191), the association between these domains needs to be stabilized by the Cys14-Cys200 disulfide bridge, whose rate of formation depends on the proximity between these two cysteine residues (Wedemeyer et al. 2000). In this scenario, shorter distances would presumably favor the formation of a stable Cys14-Cys200 disulfide bridge within an efficient time frame during the folding process of the receptor protein thereby reducing its degradation in proteasomes.

In summary, this study provides further evidence of the role of Lys191 in the EL2 of the GnRHR in defining the configuration and the association of this loop with the $\mathrm{NH}_{2}$ terminus through an interaction between residues in positions 14 and 200, which in the human receptor is mediated by formation of a disulfide bridge. In the absence of Lys191, the cell surface expression of the hGnRHR is enhanced even in the absence of the Cys14-Cys200 bridge. Our findings also offer additional information on the effects of substitutions at Cys14, overemphasizing on the importance of this particular residue in defining the tertiary structure, and function of the hGnRHR.

\section{Declaration of interest}

The authors declare that there is no conflict of interest that could be perceived as prejudicing the impartiality of the research reported.

\section{Funding}

This study was supported by grants 45991-M (to A U-A) and J49811-Q (to ÁP), from CONACyT, México, grant 2005/1/I/159 from the FOFOIInstituto Mexicano del Seguro Social, México (to A U-A), grant IN105107 from PAPIIT-UNAM, México (to Á-P), and grants HD-19899, RR-00163, HD-18185, and TW/HD-00668 from the National Institutes of Health, Bethesda, MD, USA (to P M C). Eduardo Jardón-Valadez was a post-doctoral fellow supported by CONACyT, México Eduardo Jardón-Valadez is indebted to the TEMPO group of University of California, Irvine, CA, USA, for providing computing resources.

\section{Acknowledgements}

The authors are grateful to the Dirección General de Cómputo Académico (DGSCA) of Universidad Nacional Autónoma de México (UNAM) and to the Centro de Supercomputación de Galicia (CESGA) for computing time and for their excellent service. Ángel Piñeiro thanks Xunta de Galicia for his Isidro Parga Pondal research position. Alfredo Ulloa-Aguirre is recipient of a Research Career Development Award from the Fundación-IMSS, México.

\section{References}

Arora KK, Chung HO \& Catt KJ 1999 Influence of a species-specific extracellular amino acid on expression and function of the human gonadotropin-releasing hormone receptor. Molecular Endocrinology 13 890-896.

Awara WM, Guo CH \& Conn PM 1996 Effects of Asn318 and Asp87Asn318 mutations on signal transduction by the gonadotropin-releasing hormone receptor and receptor regulation. Endocrinology 137 655-662.

Beranova M, Oliveira LM, Bedecarrats GY, Schipani E, Vallejo M, Ammini AC, Quintos JB, Hall JE, Martin KA, Hayes FJ et al. 2001 Prevalence, 
phenotypic spectrum, and modes of inheritance of gonadotropin-releasing hormone receptor mutations in idiopathic hypogonadotropic hypogonadism. Journal of Clinical Endocrinology and Metabolism 86 1580-1588.

Berendsen HJC, Postma JPM, Van Gunsteren WF, DiNola A \& Haak JR 1984 Molecular dynamics with coupling to an external bath. Journal of Chemical Physics 81 3584-3690.

Berendsen HJC, Grigera JR \& Straatsma TP 1987 The missing term in effective pair potentials. Journal of Physical Chemistry 91 6269-6271.

Berendsen HJC, van der Spoel LH \& van Drunen R 1995 GROMACS: a message-passing parallel molecular dynamics implementation. Computer Physics Communications 95 43-56.

Colson AO, Perlman JH, Smolyar A, Gershengorn MC \& Osman R 1998 Static and dynamic roles of extracellular loops in G-protein-coupled receptors: a mechanism for sequential binding of thyrotropin-releasing hormone to its receptor. Biophysical Journal 74 1087-1100.

Conn PM, Ulloa-Aguirre A, Ito J \& Janovick JA 2007 G protein-coupled receptor trafficking in health and disease: lessons learned to prepare for therapeutic mutant rescue in vivo. Pharmacological Reviews 59 225-250.

Cook JV \& Eidne KA 1997 An intramolecular disulfide bond between conserved extracellular cysteines in the gonadotropin-releasing hormone receptor is essential for binding and activation. Endocrinology $\mathbf{1 3 8}$ 2800-2806.

Chi L, Zhou W, Prikhozhan A, Flanagan C, Davidson JS, Golembo M, Illing N, Millar RP \& Sealfon SC 1993 Cloning and characterization of the human GnRH receptor. Molecular and Cellular Endocrinology 91 R1-R6.

Darden T, Darrin Y \& Pedersen L 1993 Particle mesh Ewald: an $N-\log (N)$ method for Ewald sums in large systems. Journal of Chemical Physics $\mathbf{9 8}$ 10089-10092.

Davidson JS, McArdle CA, Davies P, Elario R, Flanagan CA \& Millar RP 1996 Asn102 of the gonadotropin-releasing hormone receptor is a critical determinant of potency for agonists containing $\mathrm{C}$-terminal glycinamide. Journal of Biological Chemistry 271 15510-15514.

Davidson JS, Assefa D, Pawson A, Davies P, Hapgood J, Becker I, Flanagan C, Roeske R \& Millar R 1997 Irreversible activation of the gonadotropinreleasing hormone receptor by photoaffinity cross-linking: localization of attachment site to Cys residue in N-terminal segment. Biochemistry $\mathbf{3 6}$ 12881-12889.

Essman U, Perera L, Berkowitz ML, Darden T, Lee H \& Pedersen LG 1995 A smooth particle mesh Ewald method. Journal of Chemical Physics 103 8577-8593.

Flyvbjerg H \& Petersen HG 1989 Error estimates on averages of correlated data. Journal of Chemical Physics 91 461-466.

Heding A, Vrecl M, Bogerd J, McGregor A, Sellar R, Taylor PL \& Eidne KA 1998 Gonadotropin-releasing hormone receptors with intracellular carboxyl-terminal tails undergo acute desensitization of total inositol phosphate production and exhibit accelerated internalization kinetics. Journal of Biological Chemistry 273 11472-11477.

Hess B, Bekker H, Berendsen HJC \& Fraaije JGEM 1997 LINCS: a linear constraint solver for molecular simulations. Journal of Computational Chemistry 18 1463-1472.

Hockney RW \& Eastwood JW 1988 Computer Simulation Using Particles. Bristol, UK: Adam Hilger.

Huckle WR \& Conn PM 1987 Use of lithium ion in measurement of stimulated pituitary inositol phospholipid turnover. Methods in Enzymology 141 149-155.

Humphrey W, Dalke A \& Schulten K 1996 VMD: visual molecular dynamics. Journal of Molecular Graphics 14 33-38.

Janovick JA, Maya-Nunez G \& Conn PM 2002 Rescue of hypogonadotropic hypogonadism-causing and manufactured $\mathrm{GnRH}$ receptor mutants by a specific protein-folding template: misrouted proteins as a novel disease etiology and therapeutic target. Journal of Clinical Endocrinology and Metabolism 87 3255-3262.

Janovick JA, Goulet M, Bush E, Greer J, Wettlaufer DG \& Conn PM 2003 a Structure-activity relations of successful pharmacologic chaperones for rescue of naturally occurring and manufactured mutants of the gonadotropin-releasing hormone receptor. Journal of Pharmacology and Experimental Therapeutics 305 608-614.
Janovick JA, Ulloa-Aguirre A \& Conn PM 2003b Evolved regulation of gonadotropin-releasing hormone receptor cell surface expression. Endocrine 22 317-327.

Janovick JA, Knollman PE, Brothers SP, Ayala-Yanez R, Aziz AS \& Conn PM 2006 Regulation of $\mathrm{G}$ protein-coupled receptor trafficking by inefficient plasma membrane expression: molecular basis of an evolved strategy. Journal of Biological Chemistry 281 8417-8425.

Janovick JA, Brothers SP, Knollman PE \& Conn PM 2007 Specializations of a G-protein-coupled receptor that appear to aid with detection of frequency-modulated signals from its ligand. FASEB Journal 21 384-492.

Jardon-Valadez E, Ulloa-Aguirre A \& Pineiro A 2008 Modeling and molecular dynamics simulation of the human gonadotropin-releasing hormone receptor in a lipid bilayer. Journal of Physical Chemistry 112 10704-10713.

Knobil E 1974 On the control of gonadotropin secretion in the rhesus monkey. Recent Progress in Hormone Research 30 1-46.

Knollman PE, Janovick JA, Brothers SP \& Conn PM 2005 Parallel regulation of membrane trafficking and dominant-negative effects by misrouted gonadotropin-releasing hormone receptor mutants. Journal of Biological Chemistry $28024506-24514$.

De Lano WL 2002 The PyMOL Molecular Graphics System. In http://www.pymol.org, San Carlos, CA.

Leanos-Miranda A, Janovick JA \& Conn PM 2002 Receptor-misrouting: an unexpectedly prevalent and rescuable etiology in gonadotropin-releasing hormone receptor-mediated hypogonadotropic hypogonadism. Journal of Clinical Endocrinology and Metabolism 87 4825-4828.

Leanos-Miranda A, Ulloa-Aguirre A, Janovick JA \& Conn PM 2005 In vitro coexpression and pharmacological rescue of mutant gonadotropin-releasing hormone receptors causing hypogonadotropic hypogonadism in humans expressing compound heterozygous alleles. Journal of Clinical Endocrinology and Metabolism 90 3001-3008.

Lin X, Janovick JA, Brothers S, Blomenrohr M, Bogerd J \& Conn PM 1998 Addition of catfish gonadotropin-releasing hormone $(\mathrm{GnRH})$ receptor intracellular carboxyl-terminal tail to rat $\mathrm{GnRH}$ receptor alters receptor expression and regulation. Molecular Endocrinology 12 161-171.

Lindahl E, Hess B \& Van Der Spoel D 2001 GROMACS3.0: a package for molecular simulation and trajectory analysis. Journal of Molecular Modeling $\mathbf{7}$ 306-317.

Maya-Nunez G, Janovick JA, Ulloa-Aguirre A, Soderlund D, Conn PM \& Mendez JP 2002 Molecular basis of hypogonadotropic hypogonadism: restoration of mutant $(\mathrm{E}(90) \mathrm{K}) \mathrm{GnRH}$ receptor function by a deletion at a distant site. Journal of Clinical Endocrinology and Metabolism 87 2144-2149.

McArdle CA, Davidson JS \& Willars GB 1999 The tail of the gonadotrophinreleasing hormone receptor: desensitization at, and distal to, $G$ proteincoupled receptors. Molecular and Cellular Endocrinology 151 129-136.

Millar RP 2003 GnRH II and type II GnRH receptors. Trends in Endocrinology and Metabolism 14 35-43.

Millar RP, Lu ZL, Pawson AJ, Flanagan CA, Morgan K \& Maudsley SR 2004 Gonadotropin-releasing hormone receptors. Endocrine Reviews 25 235-275.

Miyamoto S \& Kollman PA 1992 SETTLE: an analytical version of the SHAKE and RATTLE algorithms for rigid water models. Journal of Computational Chemistry 13 952-962.

Oostenbrink C, Villa A, Mark AE \& van Gunsteren WF 2004 A biomolecular force field based on the free enthalpy of hydration and solvation: the GROMOS force-field parameter sets 53A5 and 53A6. Journal of Computational Chemistry 25 1656-1676.

Santen RJ \& Bardin CW 1973 Episodic luteinizing hormone secretion in man. Pulse analysis, clinical interpretation, physiologic mechanisms. Journal of Clinical Investigation 52 2617-2628.

Sayle RA \& Milnerwhite EJ 1995 RASMOL: biomolecular graphics for all. Trends in Biochemical Sciences 20 374-376.

Van Der Spoel D, Lindahl E, Hess B, Groenhof G, Mark AE \& Berendsen HJ 2005 GROMACS: fast, flexible, and free. Journal of Computational Chemistry 26 1701-1718 
Ulloa-Aguirre A \& Conn PM 1998 G protein-coupled receptors and the G protein family. In Handbook of Physiology. Section 7: The Endocrine System, pp 87-124. Ed. PM Conn. New York: Oxford University Press.

Ulloa-Aguirre A, Janovick JA, Brothers SP \& Conn PM 2004 Pharmacologic rescue of conformationally-defective proteins: implications for the treatment of human disease. Traffic 5 821-837.

Ulloa-Aguirre A, Janovick JA, Miranda AL \& Conn PM 2006 G-proteincoupled receptor trafficking: understanding the chemical basis of health and disease. ACS Chemical Biology 1 631-648.
Wedemeyer WJ, Welker E, Narayan M \& Scheraga HA 2000 Disulfide bonds and protein folding. Biochemistry 397032.

Received in final form 18 February 2009

Accepted 26 February 2009

Made available online as an Accepted Preprint 26 February 2009 